doi:10.1038/pr.2017.120

\section{Early career investigator}

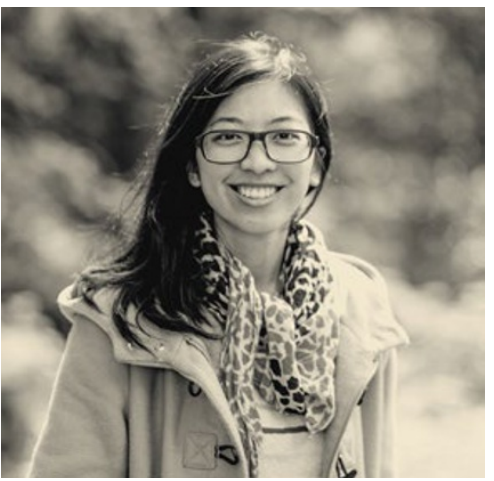

Congratulations to Theresia Handayani Mina, this issue's Early Career Investigator. She explains her motivation for a career in scientific research and describes the influence of her mentors. See page 2

\section{Perinatal inflammation is associated with morbidity}

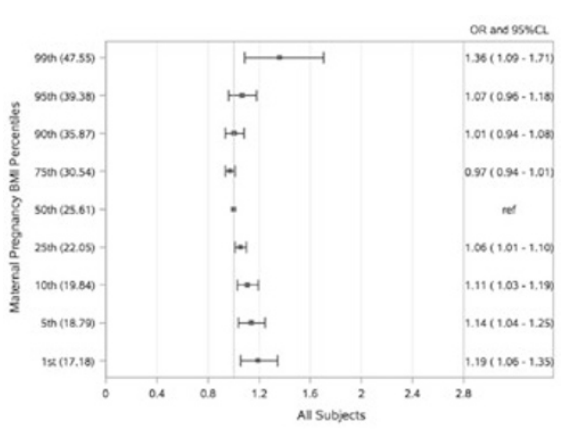

Carmichael and colleagues demonstrated in a large population study that both high and low body mass index (BMI) are associated with bronchopulmonary dysplasia (BPD). Prepregnancy BMI may influence the development of BPD by inducing an inflammatory response. See page 8

\section{Electronic cigarettes useful for smoking cessation?}

Electronic cigarettes are thought to be a useful tool for cessation of smoking. However, in a study among adolescent smokers in Hong Kong, Wang et al. found that electronic cigarette use

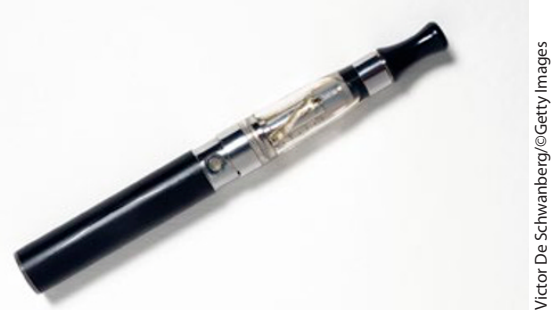

was not associated with successful smoking cessation. See page 14

\section{Cannabidiol's effects on hypoxia ischemia-induced lung injury}
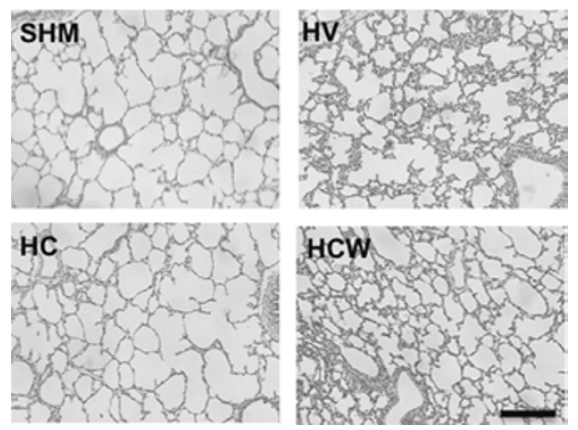

Arruza et al. showed that cannabidiol reduces lung injury in newborn piglets undergoing hypoxia-ischemia. Whether the effect of cannabidiol is on the brain or the lungs is to be determined. See page 79

\section{Effects of maternal obesity on neurodevelopment and executive function}

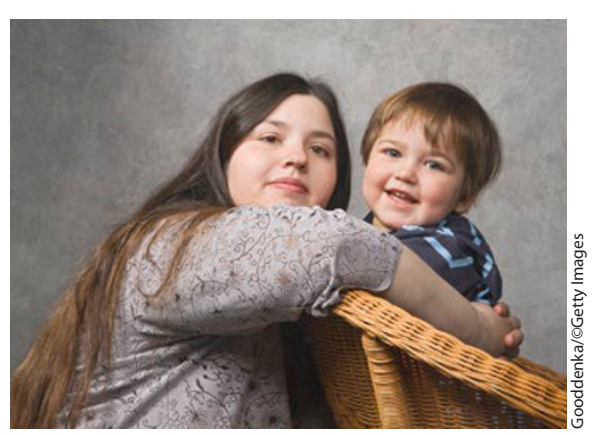

Mina and colleagues found a poorer outcome in parameters of neurocognitive function such as fine motor skills and problem solving in children born to severely obese mothers. See page 47

\section{Immunomodulatory effects of osteopontin in infant formula}

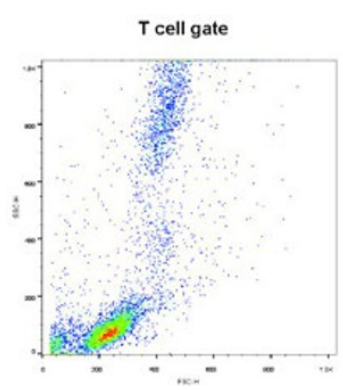

Breastfeeding is associated with a reduced risk of infections in infants. To determine whether osteopontin in breast milk is responsible for this effect, West et al. conducted a randomized human trial using formula with and without added osteopontin and observed increased proportions of T cells and monocytes with the former. Their findings may translate into important changes in formula. See page 63

\section{Postnatal intermittent hypoxia causes inflammation and white matter damage}
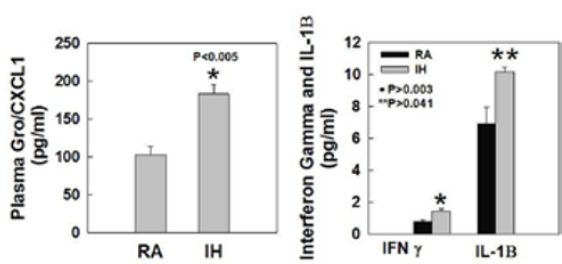

In a preterm rat model, Darnall et al. found that intermittent hypoxia caused an increase in proinflammatory cytokines both systemically and in the cerebellum. It also induced metabolic and white matter changes, as detected on magnetic resonance spectroscopy and magnetic resonance imaging, respectively. The authors conclude that postnatal hypoxia similar to that seen in preterm infants is associated with cerebral inflammation. See page 164 